\title{
The role of the bow shock in solar wind-magnetosphere coupling
}

\author{
R. E. Lopez ${ }^{1}$, V. G. Merkin ${ }^{2}$, and J. G. Lyon ${ }^{3}$ \\ ${ }^{1}$ Department of Physics, The University of Texas at Arlington, Arlington, TX, 76019, USA \\ ${ }^{2}$ The Johns Hopkins University Applied Physics Laboratory, Laurel, MD, 20723, USA \\ ${ }^{3}$ Department of Physics and Astronomy, Dartmouth College, Hanover, NH, 03755, USA
}

Received: 1 March 2011 - Revised: 4 June 2011 - Accepted: 6 June 2011 - Published: 25 June 2011

\begin{abstract}
In this paper we examine the role of the bow shock in coupling solar wind energy to the magnetosphere using global magnetohydrodynamic simulations of the solar windmagnetosphere interaction with southward IMF. During typical solar wind conditions, there are two significant dynamo currents in the magnetospheric system, one in the highlatitude mantle region tailward of the cusp and the other in the bow shock. As the magnitude of the (southward) IMF increases and the solar wind becomes a low Mach number flow, there is a significant change in solar wind-magnetosphere coupling. The high-latitude magnetopause dynamo becomes insignificant compared to the bow shock and a large load appears right outside the magnetopause. This leaves the bow shock current as the only substantial dynamo current in the system, and the only place where a significant amount of mechanical energy is extracted from the solar wind. That energy appears primarily as electromagnetic energy, and the Poynting flux generated at the bow shock feeds energy back into the plasma, reaccelerating it to solar wind speeds. Some small fraction of that Poynting flux is directed into the magnetosphere, supplying the energy needed for magnetospheric dynamics. Thus during periods when the solar wind flow has a low Mach number, the main dynamo in the solar windmagnetosphere system is the bow shock.
\end{abstract}

Keywords. Magnetospheric physics (Current systems; Magnetospheric configuration and dynamics; Solar windmagnetosphere interactions)

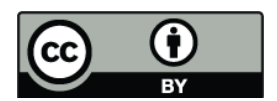

Correspondence to: R. E. Lopez (relopez@uta.edu)

\section{Introduction}

The solar wind is the ultimate source of almost all of the energy that powers the dynamics in Earth's magnetosphere, as the Earth's rotation is a minor source of power (unlike the case of Jupiter). The transfer of this energy is accomplished through two main processes. The first, and the most significant one, is magnetic merging between the interplanetary magnetic field (IMF) and the geomagnetic field (Dungey, 1961). The second (and less dynamically important) process is the viscous interaction between the magnetosphere and the solar wind flowing past it (Axford and Hines, 1961).

Typically, the rate of transfer of solar wind energy to the magnetosphere has been discussed from an electrodynamical perspective (e.g., Koskinen and Tanskanen, 2002) because the actual overall rate of energy transfer is governed primarily by dayside merging with the IMF, which in turn is highly dependent on the IMF orientation. Thus, one can view the transfer of energy in terms of the Poynting flux admitted into the magnetosphere, and the "epsilon parameter" is formulated along these lines (Akasofu, 1981). An alternative coupling function is $V B_{\mathrm{s}}$, where $B_{\mathrm{S}}$ is the southward component of the IMF and $V$ is the solar wind velocity. This coupling function is well correlated with the injection of particle energy into the ring current (Burton et al., 1975), and this relationship persists over a wide range of IMF values (Lopez et al., 2009). The magnitude of $B_{\mathrm{S}}$ is also linearly related to the transpolar potential (Boyle et al., 1997), at least for moderate IMF values (Lopez et al., 2010), and the transpolar potential is an indicator of the strength of convection in the magnetosphere. Other coupling functions have been developed either from a first principles approach that tries to quantify the dayside merging rate (Borovsky, 2008) or from empirical fits to various magnetospheric "state variables" as a function solar wind conditions (Newell et al., 2007). One can also include a separate viscous term in such coupling functions (Newell et al., 2008).

Published by Copernicus Publications on behalf of the European Geosciences Union. 


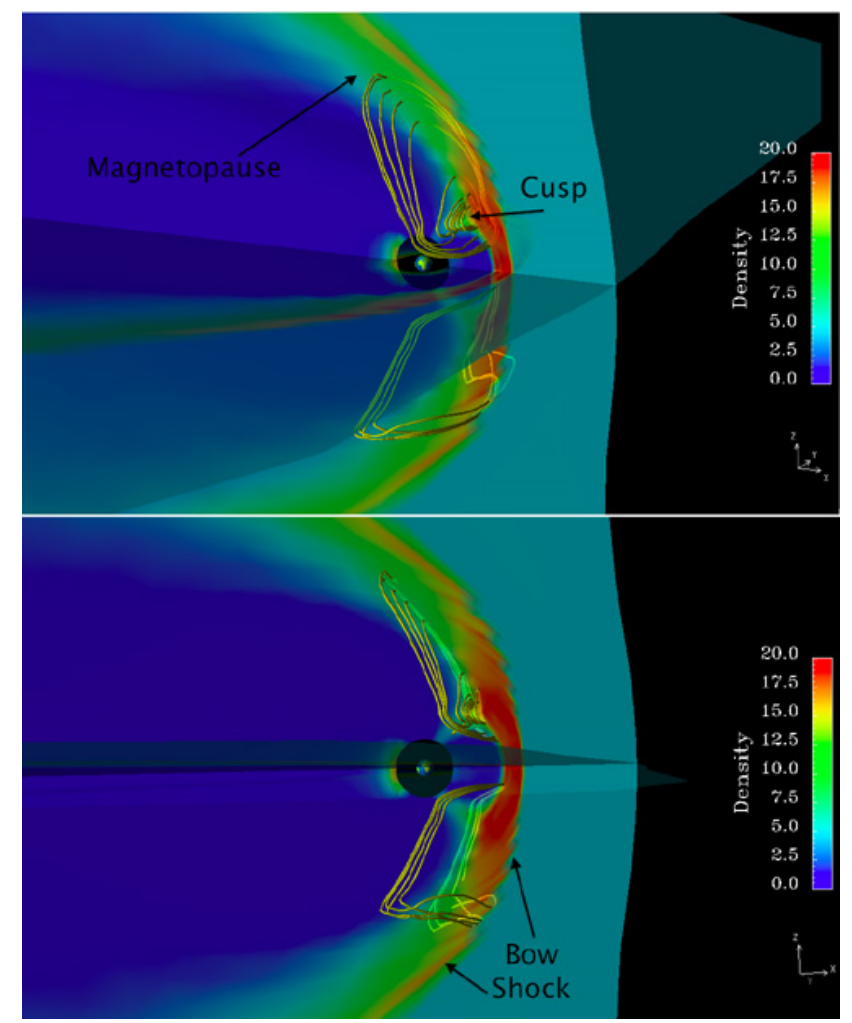

Fig. 1. Chapman-Ferraro current streamlines as determined from the LFM magnetosphere simulation under nominal solar wind conditions $\left(V=400 \mathrm{~km} \mathrm{~s}^{-1}, n=5 \mathrm{~cm}^{-3}, B_{\mathrm{Z}}=-5 \mathrm{nT}\right)$ with density $\left(\mathrm{cm}^{-3}\right)$ color-coded in the translucent XY- and XZ-planes. The GSM coordinate system is shown in the lower right corner. The sense of the current circulation is counterclockwise (as viewed from the Sun) in the Northern Hemisphere and clockwise in the Southern Hemisphere. The Northern Hemisphere current includes a portion of the cusp current, tightly wound in the center of ChapmanFerraro current system. The Southern Hemisphere current shows the connection between the bow shock current and the low latitude Chapman-Ferraro current.

However, one can also view the transfer of energy from a mechanical perspective. In the case of the viscous interaction this perspective is obvious, since the most widely proposed mechanism is the Kelvin-Helmholtz instability operating on the flanks of the magnetosphere (e.g., Otto and Fairfield, 2000; Farrugia et al., 2001). In the case of magnetic merging (for simplicity let us assume a purely southward IMF), the newly merged field lines are dragged by the flow of the solar wind, and this stress is transmitted to the ionosphere, resulting in the typical 2-cell convection pattern (e.g., Papitashvili and Rich, 2002). For northward IMF, a four-cell convection pattern is often present (Reiff and Heelis, 1994; Cumnock et al., 1995). As the overdraped fields are dragged downstream by the solar wind (Crooker, 1992), the tension produces sunward flow at the ionospheric end of the open field lines. Regardless of the orientation of the IMF, ionospheric plasma is set in motion by the stress communicated to it by the magnetic field as the solar wind drags it past the Earth. Thus the ultimate source of the energy transmitted to the magnetosphere is the mechanical energy of the solar wind, whether the mechanism of that transfer is magnetic reconnection or some other process.

In this paper we investigate just where in the system the mechanical energy of the solar wind is extracted from the flow. We utilize the Lyon-Fedder-Mobarry global MHD model (Lyon et al., 2004) to examine where that energy is extracted from the solar wind flow and where it is deposited under the condition of a purely southward IMF. We will demonstrate that when the (southward) IMF is very large, the primary place in the system where solar wind mechanical energy is extracted is at the bow shock, and that dynamo is the source of the solar wind energy that is delivered to the magnetosphere.

\section{The Chapman-Ferraro magnetosphere}

Under normal circumstances with nominal solar wind conditions (speed $\sim 400 \mathrm{~km} \mathrm{~s}^{-1}$, density $\sim 5 \mathrm{~cm}^{-3}$, IMF $\sim$ a few $\mathrm{nT}$ ), the major force standing off the solar wind is the $\boldsymbol{J} \times \boldsymbol{B}$ force from the Chapman-Ferraro current. This current at low latitudes runs from dawn to dusk, as is required by the compression of the magnetospheric magnetic field. At high latitudes, in the plasma mantle poleward of the cusp, the magnetic shear, and hence the direction of the current, reverses. Thus, at the most basic level, the Chapman-Ferraro currents close on themselves on the magnetopause, flowing dawn to dusk at low latitude and dusk to dawn at high latitude. This current system is illustrated in Fig. 1 using results from the LFM simulation code for a case with purely southward IMF, and the magnetopause currents in the Northern Hemisphere show the pattern very clearly.

Siebert and Siscoe (2002) examined the question of energy extraction and deposition using the framework of circuits, identifying dynamos and loads in the system as simulated by a global MHD code. They point out that MHD codes operate by implementing Newton's Laws and Maxwell's Equations, so the results the codes produce are consistent with the primacy of the inertia of the solar wind plasma in determining the dynamics of the situation (e.g., Vasyliunas, 2001). However, the dynamo framework does provide a means for analyzing the MHD results post hoc to determine the energy sources and sinks in the system. In particular, Siebert and Siscoe (2002) argue, based on evidence from MHD simulations, that the dynamo that powers the low latitude ChapmanFerraro current and reconnection at the dayside merging line is actually the bow shock.

A dynamo current is one in which $\boldsymbol{J} \bullet \boldsymbol{E}<0$, whereas a load has $\boldsymbol{J} \bullet \boldsymbol{E}>0$. For southward IMF, the solar wind electric field in the frame of the Earth is directed in the positive Y-direction. Using LFM simulation results for nominal solar 


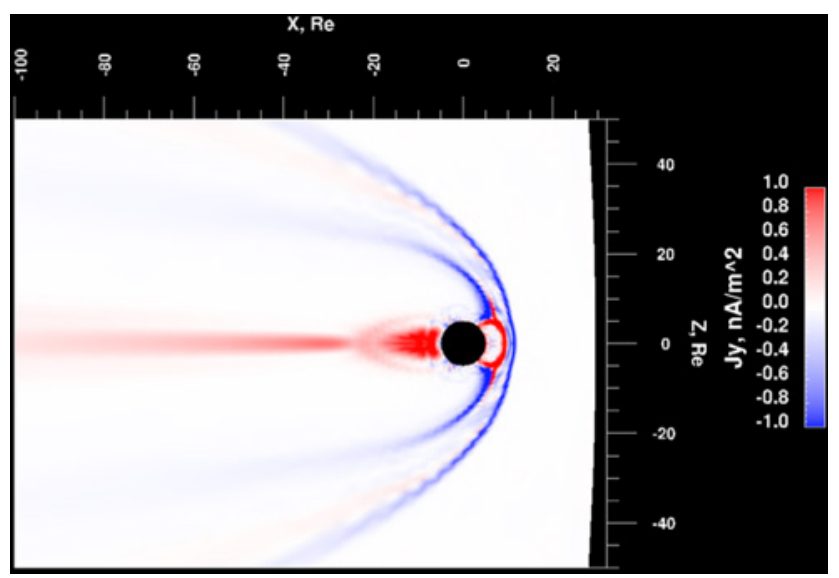

Fig. 2. Distribution of $J_{\mathrm{y}}$ in the meridional plane as determined from the LFM magnetosphere simulation under nominal solar wind conditions with purely southward IMF $\left(V=400 \mathrm{~km} \mathrm{~s}^{-1}\right.$, $\left.n=5 \mathrm{~cm}^{-3}, B_{\mathrm{Z}}=-5 \mathrm{nT}\right)$.

wind conditions, Fig. 2 presents $J_{\mathrm{y}}$ in the meridional plane, and so dynamo currents are located where $J_{\mathrm{y}}$ is negative and loads are located where $J_{\mathrm{y}}$ is positive. It is evident that the low-latitude Chapman-Ferraro current is a load, while the high-latitude mantle current is a dynamo. In addition, the entire bow shock contains a dynamo current.

Figure 1 actually contains two current systems, illustrated by two sets of current streamlines, and it resembles Fig. 1 from Siebert and Siscoe (2002). The Northern Hemisphere current streamline that was initiated not far from the cusp illustrates the standard Chapman-Ferraro current that closes along the boundary. The portion of the current equatorward of the cusp flows from dawn to dusk across noon and constitutes a load, whereas the current poleward of the cusp flows in the opposite direction across the noon-midnight meridional plane. Thus the high latitude currents poleward of the cusp constitute a dynamo system where solar wind mechanical energy is extracted and transported as Poynting flux to lower latitudes.

On the other hand, the low latitude, equatorial current, which we would identify as the current passing through the dayside merging region, connects to the bow shock, as surmised by Siebert and Siscoe (2002). This can be seen by inspecting the current streamlines in the Southern Hemisphere in Fig. 1. The southern current streamline was initiated near the equatorial plane at the noon magnetopause. The current streamline does not stay on the magnetopause (in contrast to current streamline in the Northern Hemisphere), and one can see that the current streamline passes through the magnetosheath and connects to the bow shock. In fact, any current streamline that is started near the region where one would expect the merging line to be located connects to the bow shock. Thus, we agree with the Siebert and Siscoe (2002) that the equatorial magnetospheric load is connected to the bow shock current dynamo. However, the presence of an extensive high-latitude dynamo means that a significant portion of the power consumed by the dayside Chapman-Ferraro current overall is generated on the magnetopause itself.

\section{Large IMF, the saturation of the potential, and the bow shock}

Typically the strength of magnetospheric convection, as indicated by the magnitude of the transpolar ionospheric potential, responds linearly to the magnitude of the southward component of the IMF (e.g., Boyle et al., 1997). However, when the southward component of the IMF becomes very large, the potential behaves in a non-linear fashion, reaching a maximum value that is then fairly insensitive to further increases in IMF magnitude (e.g., Russell et al., 2001).

A number of explanations for this effect have been offered (Siscoe et al., 2002a, b; Merkin et al., 2005; Ridley, 2007; Kivelson and Ridley, 2008; Lopez et al., 2010), and Borovsky et al. (2009) conducted an extensive study of the behavior of a simulated magnetosphere under saturation conditions. However, only one of these models (Lopez et al., 2010) explains the dependence of the transpolar potential on solar wind parameters during both saturation conditions and normal IMF conditions by means of a single mechanism. Regardless of the specific mechanism that is invoked to explain saturation, all of these models limit the rate at which flux crosses the dayside merging line, which (in the absence of field-aligned potential drops) is identically equal to the transpolar potential.

The solar wind conditions for saturation constitute a low Alfvén Mach number solar wind flow. Under such conditions, the solar wind-magnetosphere interaction becomes significantly altered (Lavraud and Borovsky, 2008). For example, under the normal conditions of high Mach number flow, the bow shock compression ratio is close to the theoretical maximum of 4 , and it is not particularly sensitive to solar wind conditions. However, when the Mach number is low, the compression ratio does vary significantly as a function of upstream conditions. By changing the compression ratio, large effects in the magnetosheath can result from solar wind variations that would normally have little effect on magnetosheath dynamics. Since it is the magnetosheath that is actually in contact with the magnetopause, variations in solar wind density during periods of large southward IMF are directly reflected in the transfer of energy to the magnetosphere (Lopez et al., 2004).

Another feature of low Mach number flows is that the magnetosheath becomes magnetically dominated $(\beta<1)$, so that the $\boldsymbol{J} \times \boldsymbol{B}$ force is significantly larger than the $-\nabla P$ force that usually controls the magnetosheath flow (Chen et al., 1993; Lavraud and Borovsky, 2008; Lopez et al., 2010). Figure 3 presents LFM simulation results for a period with $V=400 \mathrm{~km} \mathrm{~s}^{-1}, n=5 \mathrm{~cm}^{-3}$, and $B_{\mathrm{z}}=-20 \mathrm{nT}$ 


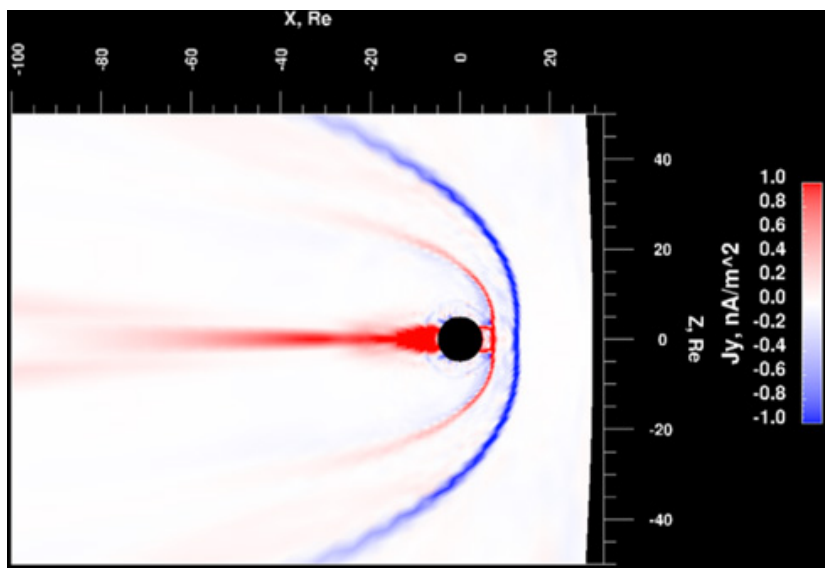

Fig. 3. Distribution of $J_{\mathrm{y}}$ in the meridional plane as determined from the LFM magnetosphere simulation under solar wind conditions that produce saturation with purely southward IMF $(V=$ $\left.400 \mathrm{~km} \mathrm{~s}^{-1}, n=5 \mathrm{~cm}^{-3}, B_{\mathrm{Z}}=-20 \mathrm{nT}\right)$.

(purely southward IMF). The high latitude current in Fig. 3 (which in Fig. 2 was directed in the negative Y-direction) appears to have reversed direction and now flows in the positive Y-direction, the same direction as the low latitude magnetopause current. Actually, that current is in the magnetosheath, not on the magnetopause, and, since it is a load current, we suspect that it is associated with the acceleration and diversion of the plasma flow around the magnetospheric obstacle by the dominant $\boldsymbol{J} \times \boldsymbol{B}$ force. There is an additional weak current (blue) that does flow on the magnetopause, and it is the remnant of the original mantle dynamo system.

In contrast to Fig. 2, there is almost no high-latitude mantle dynamo and instead we see a load all along the highlatitude region that is much bigger than the remnant mantle generator. The bow shock, however, remains a dynamo. In fact, under these conditions it is the only place in the geospace system where substantial solar wind mechanical energy is extracted from the flow. Thus, the bulk of the solar wind mechanical energy that powers magnetospheric processes, as well as the diversion and acceleration of the magnetospheath flow, must be transported from the bow shock where it is extracted from the solar wind flow. Since the energy is primarily magnetic, this transport must be accomplished by a large Poynting flux from the bow shock into the magnetosheath, some fraction of which enters the magnetosphere.

This result is not unique to the LFM simulation. Figure 4 shows results from the University of Michigan BATSRUS code (Powell et al., 1999) run at the Community Coordinated Modeling Center (the images were created on the CCMC site). The simulations were run with the same solar wind conditions as the LFM simulations discussed above $\left(V=400 \mathrm{~km} \mathrm{~s}^{-1}, n=5 \mathrm{~cm}^{-3}\right)$, with purely southward IMF and a uniform Pedersen conductance in the ionosphere. The

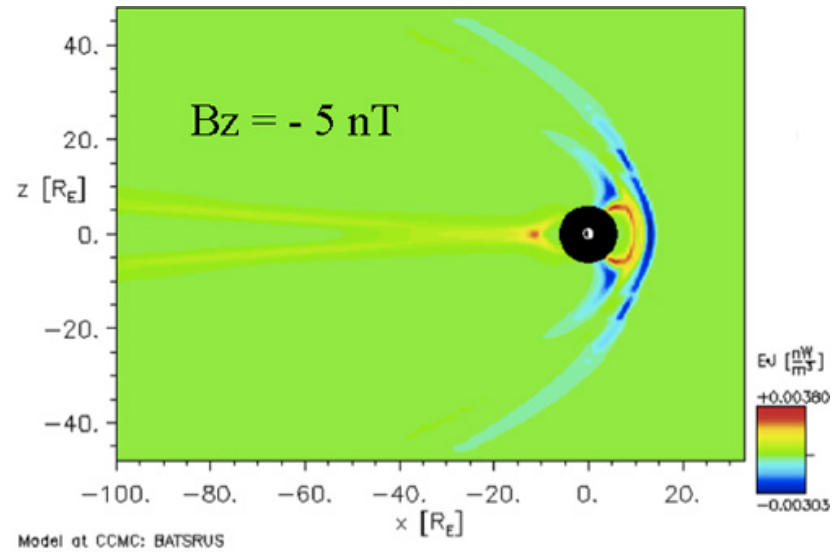

01/01/2000 Time $=02: 00: 00$ UT $y=0.00 R_{t}$

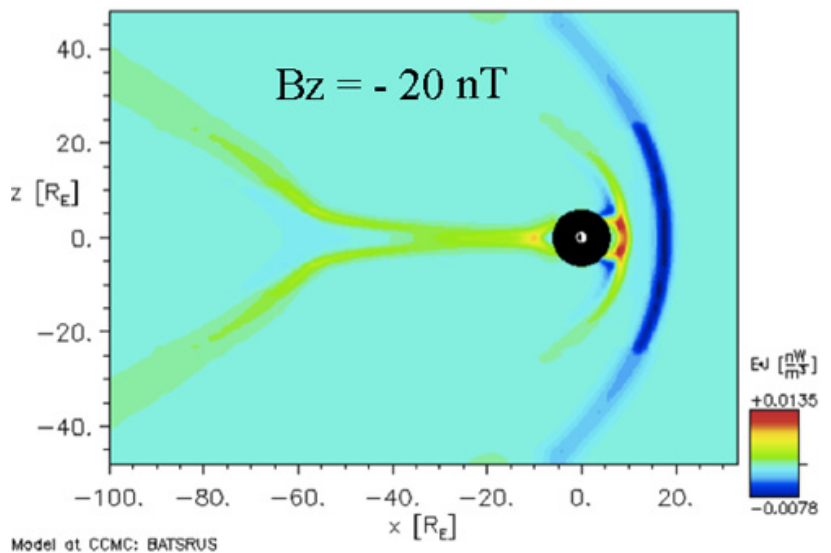

Fig. 4. Distribution of $\boldsymbol{J} \bullet \boldsymbol{E}$ in the meridional plane from BATSRUS simulations for solar wind with $V=400 \mathrm{~km} \mathrm{~s}^{-1}, n=5 \mathrm{~cm}^{-3}$ and purely southward IMF.

$-5 \mathrm{nT}$ run shows a large mantle dynamo region, while the $-20 \mathrm{nT}$ run (when the potential is saturated) shows a much smaller mantle dynamo and the additional magnetosheath load. Moreover, the high-latitude dynamo appears to be caused by breaking of the reconnection outflow jet as opposed to slowing of solar wind/magnetosheath flow. Thus, both simulation codes lead us to the same result. During large southward IMF the bow shock is by far the dominant dynamo in the system.

A second point to notice is that the low latitude magnetopause current in Fig. 3 appears to be weaker than that in Fig. 2, which would indicate a relatively smaller compression of the dayside field. Siscoe et al. (2002a) argue that the Chapman-Ferraro current disappears entirely during saturation for strong southward IMF, which is not the case in the simulation results presented here. But the low latitude magnetopause current that one finds is smaller than that in the typical magnetosphere, and certainly during such solar wind intervals the dayside geosynchronous magnetic field can often be weaker than the dipole value (Borovsky et al., 
2009), indicating that the compression in the field from the Chapman-Ferraro current is a smaller contribution than the reduction of the dayside field associated with the Region 1 currents (e.g., Sibeck et al., 1991; Wiltberger et al., 2003).

Siscoe et al. (2002a) also posit that the Region 1 currents are the ones that produce the force that stands off the solar wind, and that these currents are closed in part by the bow shock current. In fact, for the Region 1 current system to exert an outward force on a solar wind with southward IMF, the current must flow from west to east, which is the direction of the bow shock current. The bow shock current is the only one that can provide Region 1 closure since almost all of the current flowing through the magnetosheath and on the magnetopause has the same polarity as Region 1 (east to west), so the return current must be somewhere else. LFM simulations do show that the major part of the force balance with the solar wind can take place at the bow shock if the IMF is large enough, but the bow shock current does not completely supplant the Chapman-Ferraro current (Lopez et al., 2010), which is consistent with Fig. 3.

With regard to a portion of the bow shock current closing through the Region 1 Birkeland currents, there is evidence that a significant amount of Region 1 current extends into the polar cap, flowing on open field lines, during periods with large southward IMF (Lopez et al., 2008). Birkeland currents flowing on open field lines in the polar cap must close somewhere, and the most reasonable place for that to be is in the magnetosheath. The magnetosheath is full of current throughout the volume (not just tracing current streamlines) that has Region 1 polarity and which closes on the bow shock (Lopez et al., 2010). Therefore, we find substantial evidence in favor of the arguments advanced by Siscoe et al. (2002a).

\section{Discussion}

The bow shock current itself is due to the shear in the magnetic field due to the compression of the field across the shock. This shear is driven by the energy that is extracted from the flow and put into the field, and this electromagnetic energy is transported as Poynting flux across the magnetosheath. In the MHD approximation, the direction of the Poynting flux is the same as the plasma velocity perpendicular to the magnetic field. Figure 5 illustrates the overall distribution of the flow, the force on the flow, and the magnetic shear (current) throughout the magnetosheath for a strongly southward IMF, such as the conditions used for the simulation in Fig. 3.

The flow in the magnetosheath is primarily around the magnetospheric obstacle, and so too is the energy flow. Only a small portion of the flow actually crosses the merging line, delivering magnetic flux and energy to the magnetosphere. The limited Y-extent of the solar wind flow that actually crosses the merging line is known as the geoeffective length (Burke et al., 1999; Lopez et al., 2010). Most of the energy

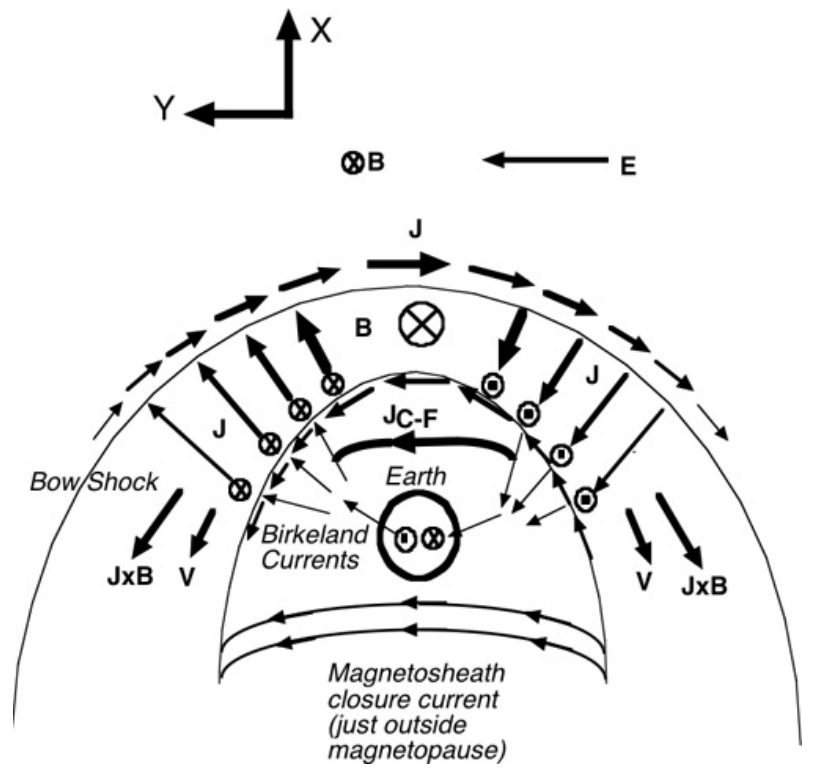

Fig. 5. A schematic for current closure through the geospace system for conditions of large purely southward IMF as viewed from above the north pole.

extracted from the solar wind at the bow shock is deposited back into the plasma as the magnetosheath $\boldsymbol{J} \times \boldsymbol{B}$ reaccelerates the flow to solar wind velocity.

The maximum compression of the IMF is at the nose of the bow shock, and that is where the bow shock current is strongest. Away from the nose the compression is less and the bow shock current must be less. The diversion of the bow shock current is carried through the magnetosheath, and that closure current by necessity has Region 1 polarity for southward IMF. Near the equatorial plane, this current is the current that closes the low-latitude Chapman-Ferraro current, so that the bow shock dynamo can supply energy to the load on the merging line as discussed by Siebert and Siscoe (2002). These features are always there for southward IMF, regardless of the magnitude of the IMF.

The high-latitude magnetopause, however, has a fundamentally different structure when the magnetosheath is magnetically dominated. The total high-latitude current seen in Figs. 1 and 2 has reversed direction, as seen in Fig. 3. Now the current flows across the magnetopause/magnetosheath from dawn to dusk (Region 1 sense) and that helps to close the bow shock current in the magnetosheath. So in this case, a small amount of the magnetopause current actually closes on the magnetopause, but the bulk of it must close through the magnetosheath to the bow shock. In addition, some of the magnetosheath current closes directly into the polar ionosphere, as discussed above. The ionosphere is also a load, and so some of the energy dissipated in the polar cap is Poynting flux coming directly from the bow shock that is not processed through the intermediary of dayside merging (Lopez et al., 2010). 
The basic physics discussed here does not qualitatively depend on the orientation of the IMF, even though all of the simulation results presented above were for purely southward IMF. Recent studies have demonstrated that the transpolar potential saturates for large northward IMF (Wilder et al., 2008) and large magnitude $B_{\mathrm{y}}$ IMF as well (Mitchell et al., 2010). The saturation potential values are lower values than the saturation value of the potential for southward IMF, as one would expect from less efficient reconnection. However, the value of the IMF magnitude at which saturation effects occur is the same regardless of the direction of the IMF. A low Mach number flow and the transition to a magnetically dominated magnetosheath simply depends on having a large enough IMF magnitude, not its direction.

Consider a situation with strongly northward IMF. The current across the bow shock reverses direction compared to the direction illustrated in Fig. 5. So the diversion of the bow shock current and the corresponding shear in the magnetosheath must also reverse direction. Thus, the magnetosheath current will have NBz polarity, toward Earth at dusk and away from Earth at dawn. But since the sign of $B_{\mathrm{Z}}$ in the sheath is now positive, $\boldsymbol{J} \times \boldsymbol{B}$ stays pointing in the direction needed to deflect the magnetosheath flow around the magnetospheric obstacle and reaccelerate it. Thus for large northward IMF, one could draw a figure like Fig. 5 by simply reversing the direction of the magnetic shear (current) throughout the magnetosheath (consistent with the NBz current system) and on the magnetopause, although the direction of $\boldsymbol{J} \times \boldsymbol{B}$ in the magnetosheath would not change, nor would the fact that the bow shock is the only significant dynamo current in the system.

In general, all low Alfvén Mach number shocks in space plasmas have the same features as discussed here. There will be a very large current on the shock, and a large amount of flow energy will be converted into electromagnetic energy, as opposed to the case in high Mach number shocks were the downstream energy is dominated by plasma thermal energy. This has some interesting implications. First, where and how do these large astrophysical current systems close? In the case of an obstacle producing the shock, the currents must close on the object producing the shock, as in the case of the Earth's magnetosphere. Depending on the amount of interconnection between the field of the object and the external field, there will be a direct access of Poynting flux to the object on open field lines, as well as a transfer of electromagnetic energy to any load current systems, such as a reconnection region. What about the case of a free shock in space, such as in corotating interaction regions, or in an expanding supernova remnant? Those shocks will also convert flow energy into electromagnetic energy, and that energy must be deposited somewhere, wherever the shock currents close and drive a load. Thus a supernova remnant shock may be depositing electromagnetic energy extracted from the flow energy into loads far from the shock itself.

\section{Conclusions}

The role of the bow shock in magnetospheric dynamics has generally been unappreciated. Under nominal solar wind conditions there is little variation in the shock strength with solar wind variations, the dominant conversion of energy is from plasma flow to plasma thermal energy, and the question of the closure of the bow shock current has not been viewed as a significant issue (at least until Siebert and Siscoe, 2002). However, under conditions of a low Mach number solar wind flow, the magnetosphere enters a new dynamical regime, characterized by the saturation of the transpolar ionospheric potential. The high latitude magnetopause current dynamo becomes insignificant and a large load appears in the magnetosheath right outside the magnetopause. This means that the bow shock will be the dominamt dynamo current in the system, and almost all of the extraction of solar wind mechanical energy occurs at the bow shock. This energy must then be transported to the magnetosheath and magnetosphere. Finally, this study raises the general question of current closure in astrophysical shocks, and where the electromagnetic energy converted from mechanical energy extracted at the shock is deposited.

Acknowledgements. This paper is based upon work supported by CISM, which is funded by the STC Program of the National Science Foundation under agreement ATM-0120950, NASA grant NNX09AI63G, and NSF grant ATM-0900920. We thank the CCMC for providing users with the facility to explore MHD simulation runs and visualize the results and Robert Bruntz for helpful comments on the manuscript.

Guest Editor M. Balikhin thanks D. Sibeck and another anonymous referee for their help in evaluating this paper.

\section{References}

Akasofu, S.-I.: Energy coupling between the solar wind and the magnetosphere, Space Sci. Rev., 28, 121-190, 1981.

Axford, W. I. and Hines, C. O.: A unifying theory of high-latitude geophysical phenomena and geomagnetic storms, Can. J. Phys., 39, 1433-1464, 1961.

Borovsky, J. E.: The rudiments of a theory of solar wind/magnetosphere coupling derived from first principles, J. Geophys. Res., 113, A08228, doi:10.1029/2007JA012646, 2008.

Borovsky, J. E., Lavraud, B., and Kuznetsova, M. M.: Polar cap potential saturation, dayside reconnection, and changes to the magnetosphere, J. Geophys. Res., 114, A03224, doi:10.1029/2009JA014058, 2009.

Boyle, C. B., Reiff, P. H., and Hairston, M. R.: Empirical polar cap potentials, J. Geophys. Res., 102, 111-126, 1997.

Burke, W. J., Weimer, D. R., and Maynard, N. C.: Geoeffective inter- planetary scale sizes derived from regression analysis of polar cap potentials, J. Geophys. Res., 104(A5), 9989-9994, doi:10.1029/1999JA900031, 1999.

Burton, R. K., McPherron, R. L., and Russell, C. T.: An empirical relationship between interplanetary conditions and Dst, J. Geophys. Res., 80, 4204-4214, 1975. 
Chen, S.-H., Kivelson, M., Gosling, J., Walker, R., and Lazarus, A.: Anomalous Aspects of Magnetosheath Flow and of the Shape and Oscillations of the Magnetopause During an Interval of Strongly Northward Interplanetary Magnetic Field, J. Geophys. Res., 98(A4), 5727-5742, 1993.

Crooker, N. U.: Reverse Convection, J. Geophys. Res., 97(A12), 19363-19372, 1992.

Cumnock, J. A., Heelis, R. A., Hairston, M. R., and Newell, P. T.: High-Latitude Ionospheric Convection Pattern During Steady Northward Interplanetary Magnetic Field, J. Geophys. Res., 100(A8), 14537-14555, 1995.

Dungey, J. W.: Interplanetary magnetic field and the auroral zones, Phys. Rev. Lett., 6, 47-49, 1961.

Farrugia, C. J., Fausto, T., Gratton, T., and Torbert, R. B.: Viscous-type processes in the solar wind-magnetosphere interaction, Space Science Reviews, 95, 443-456, doi:10.1023/A:1005288703357, 2001.

Kivelson, M. G. and Ridley, A. J.: Saturation of the polar cap potential: Inference from Alfvén wing arguments, J. Geophys. Res., 113, A05214, doi:10.1029/2007JA012302, 2008.

Koskinen, H. E. J. and Tanskanen, E.: Magnetospheric energy budget and the epsilon parameter: J. Geophys. Res., 107(A11), 1415, doi:10.1029/2002JA009283, 2002.

Lavraud, B. and Borovsky, J. E.: Altered solar wind-magnetosphere interaction at low Mach numbers: Coronal mass ejections, J. Geophys. Res., 113, A00B08, doi:10.1029/2008JA013192, 2008.

Lopez, R. E., Wiltberger, M., Hernandez, S., and Lyon, J. G.: Solar wind density control of energy transfer to the magnetosphere, Geophys. Res. Lett., 31, L08804, doi:10.1029/2003GL018780, 2004.

Lopez, R. E., Hernandez, S., Hallman, K, Valenzuela, R., Seiler, J., Anderson, P., and Hairston, M.: Field-Aligned Currents in the Polar Cap during Saturation of the Polar Cap Potential, J. Atmos. Sol. Terr. Phys., 70, 555-563, doi:10.1016/j.jastp.2007.08.072, 2008.

Lopez, R. E., Lyon, J. G., Mitchell, E., Bruntz, R., Merkin, V. G., Brogl, S., Toffoletto, F., and Wiltberger, M.: Why doesn't the ring current injection rate saturate?, J. Geophys. Res., 114, A02204, doi:10.1029/2008JA013141, 2009.

Lopez, R. E., Bruntz, R., Mitchell, E. J., Wiltberger, M., Lyon, J. G., and Merkin, V. G.: The role of magnetosheath force balance in regulating the dayside reconnection potential, J. Geophys. Res., 115, A12216, doi:10.1029/2009JA014597, 2010.

Lyon, J. G., Fedder, J. A., and Mobarry, C. M.: The LyonFedder-Mobarry (LFM) global MHD magnetospheric simulation code, J. Atmos. Sol. Terr. Phys., 66, 1333-1350, doi:10.1016/j.jastp.2004.03.020, 2004.

Merkin, V., Sharma, A. S., Papadopoulos, K., Milikh, G., Lyon, J., and Goodrich, C.: Global MHD simulations of the strongly driven magnetosphere: Modeling of the transpolar potential saturation, J. Geophys. Res., 110, A09203, doi:10.1029/2004JA010993, 2005.

Mitchell, E. J., Lopez, R. E., Bruntz, R., Wiltberger, M., Lyon, J. G., Allen, R. C., Cockrell, S. J., and Whittlesey, P. L.: Saturation of Transpolar Potential for Large Y-component Interplanetary Magnetic Field, J. Geophys. Res., 115, A06201, doi:10.1029/2009JA015119, 2010.
Newell, P. T., Sotirelis, T., Liou, K., Meng, C.-I., and Rich, F. J.: A nearly universal solar wind-magnetosphere coupling function inferred from 10 magnetospheric state variables, J. Geophys. Res., 112, A01206, doi:10.1029/2006JA012015, 2007.

Newell, P. T., Sotirelis, T., Liou, K., and Rich, F. J.: A nearly universal solar Pairs of solar wind-magnetosphere coupling functions: Combining a merging term with a viscous term works best, J. Geophys. Res., 113, A04218, doi:10.1029/2007JA012825, 2008.

Otto, A. and Fairfield, D. H.: Kelvin-Helmholtz instability at the magnetotail boundary: MHD simulation and comparison with Geotail observations, J. Geophys. Res., 105(A9), 21175-21190, 2000.

Papitashvili, V. O. and Rich, F. J.: High-latitude ionospheric convection models derived from Defense Meteorological Satellite Program ion drift observations and parameterized by the interplanetary magnetic field strength and direction, J. Geophys. Res., 107(A8), 1198, doi:10.1029/2001JA000264, 2002.

Powell, K., Roe, P., Linde, T., Gombosi, T., and De Zeeuw, D. L.: A solution-adaptive upwind scheme for ideal magnetohydrodynamics, J. Comp. Phys., 154, 284-309, 1999.

Reiff, P. and Heelis, R.: Four Cells or Two? Are Four Convection Cells Really Necessary?, J. Geophys. Res., 99(A3), 3955-3959, 1994.

Ridley, A. J.: Alfvén wings at Earth's magnetosphere under strong interplanetary magnetic fields, Ann. Geophys., 25, 533-542, doi:10.5194/angeo-25-533-2007, 2007.

Russell, C. T., Luhmann, J. G., and Lu, G.: Nonlinear response of the polar ionosphere to large values of the interplanetary electric field, J. Geophys. Res., 106, 18495-18504, doi:10.1029/2001JA900053, 2001.

Sibeck, D. G., Lopez, R. E., and Roelof, E. C.: Solar wind control of magnetopause shape, location, and motion, J. Geophys. Res., 96, 5489-5495, 1991.

Siebert, K. D. and Siscoe, G. L.: Dynamo circuits for magnetopause reconnection, J. Geophys. Res., 107(A7), 1095, doi:10.1029/2001JA000237, 2002.

Siscoe, G. L., Crooker, N. U., and Siebert, K. D.: Transpolar potential saturation: Roles of Region 1 current system and solar wind ram pressure, J. Geophys. Res., 107(A10), 1321, doi:10.1029/2001JA009176, 2002a.

Siscoe, G. L., Erickson, G. M., Sonnerup, B. U. O., Maynard, N. C., Schoendorf, J. A., Siebert, K. D., Weimer, D. R., White, W. W., and Wilson, G. R.: Hill model of transpolar potential saturation: Comparisons with MHD simulations, J. Geophys. Res., 107(A6), 1075, doi:10.1029/2001JA000109, 2002b.

Vasyliunas, V. M.: Electric field and plasma flow: What drives what?, Geophys. Res. Lett., 28, 2177-2180, 2001.

Wilder, F. D., Clauer, C. R., and Baker, J. B. H.: Reverse convection potential saturation during northward IMF, Geophys. Res. Lett., 35, L12103, doi:10.1029/2008GL034040, 2008.

Wiltberger, M., Lopez, R. E., and Lyon, J. G.: Magnetopause erosion: A global view from MHD simulations, J. Geophys. Res., 108(A6), 1235, doi:10.1029/2002JA009564, 2003. 